$$
\left\{\begin{array}{c}
\frac{d}{d t}\left(\Delta^{(m-1)} q_{s r}\right)-\varepsilon F_{m}^{\prime}\left(t, q_{s r}, \dot{q}_{s r}, \cdots, q_{s r}\right), \\
\frac{d}{d t}(\Delta t)-\varepsilon f_{m}\left(t, q_{s r}, \dot{q}_{s r}, \cdots, q_{s r}\right) \\
(s-1,2, \cdots, n),
\end{array}\right.
$$

可以得到

$$
\delta g_{s r}^{(m)}-\varepsilon\left[F_{m}^{s}-{\left.\stackrel{(m)}{q_{s}} f_{m}\right],}^{(m)}\right.
$$

于是我们得到如下定理:

只要无穷小变换的生成函数 $F_{m}^{\prime} f_{m}$ 以及 规范函数 $P$ 满足

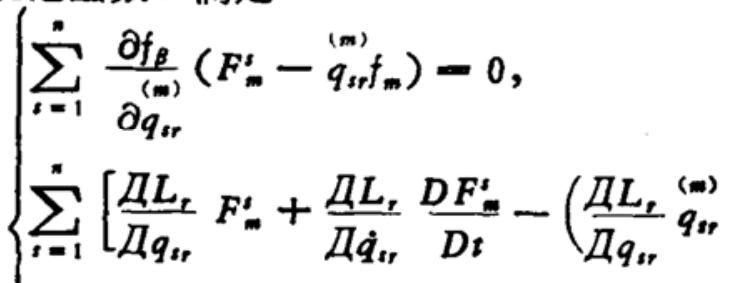

$$
\begin{aligned}
& \left.+\frac{\not L_{r}(m+1)}{\bar{Z} q_{s r}} q_{s r}\right) f_{m}-\frac{\not L L_{r}}{\bar{\partial} q_{s r}} \stackrel{(m)}{q r}^{\frac{D f_{m}}{D t}}+\left(Q^{\prime}\right.
\end{aligned}
$$

$$
\left[\begin{array}{l}
\left.+Q_{s}^{b}+\Gamma_{s}+\psi_{s}\right)\left(F_{m}^{s}-\stackrel{(m)}{\left.q_{s s} f_{m}\right)}\right]-\frac{D P}{D t} \\
=0,
\end{array}\right.
$$

则变质量任意阶非完整非保守力学系统相对 于非惯性系的运动存在下列守恒量

$$
\sum_{s=1}^{n} \frac{\not L_{r}}{\bar{Д}_{\dot{q}} r}\left(F_{m}^{s}-{\left.\stackrel{(m)}{q_{s r}} f_{m}\right)}\right)-P-\text { const. }
$$

本文的结果具有普遍性, 以往的Noether 定理都可由此定理得到。

\section{考文献}

[1] 刘端,力学学报, 1989, 1: 75-83.

[2]张解放,科学通报, 34(1989),22: 1756-1757.

罗 绍 凯

（商丘师范专科学校,商丘 476000)

\title{
原子簇 $\mathrm{C}_{60}, \mathrm{C}_{60} \mathrm{H}_{60}$ 和 $\mathrm{C}_{60} \mathrm{~F}_{60}$ 电子结构研究
}

近年来, 用激光汽化石表固体,导致非寻 常碳原子簇 $\mathrm{C}_{38}, \mathrm{C}_{50}, \mathrm{C}_{60}, \cdots, \mathrm{C}_{120}$ 的生成. 质 谱分析表明以 $\mathrm{C}_{60}$ 为主要成分. 它具 $I_{\Delta}$ 群对 称性并且最稳定 ${ }^{\omega}$. 此外, 人们还合成了一 些有实用价值的 $\mathrm{C}_{60}$ 行生物, 如 $\mathrm{C}_{60} \mathrm{Fe}$ 和 $C_{60} \mathrm{~F}_{60}$ 等.

鉴于这类化合物的特殊结构及重要实用 价值, 人们已对 $\mathrm{C}_{60}$ 开展了一些理论研究 ${ }^{[2-6]}$. 结论是足球式比手臂式结构更稳定. 例如, INDO 计算指出前者比后者稳定 $94.423 \mathrm{eV}^{[4]}$. 本文用新近在 Micro Vax II 机上扩大的 CNDO/2 程序完成了 $\mathrm{C}_{60}, \mathrm{C}_{60} \mathrm{H}_{60}$ 和 $\mathrm{C}_{60} \mathrm{~F}_{60}$ 的 系统研究. 为认识此类化合物提供了有益的 结构信息.

首先对被切割的二十面体结构（足球式） $\mathrm{C}_{60}$ 做了粗略几何优化, 得到优化的边长 $a=$ $1.421 \AA$. 固定 $\gamma_{\mathrm{CC}}$ 为此值, 选 $\gamma_{\mathrm{CH}}-1.08 \AA$ 和 $\gamma_{C P}-1.385 \AA$ 分别完成了 $\mathrm{C}_{60} \mathrm{H}_{60}$ 和 $\mathrm{C}_{60} \mathrm{~F}_{60}$
电子结构计算. 从分子总能 量值 可得到 $\mathrm{C}_{60} \mathrm{H}_{60}$ 相对于 $\mathrm{C}_{60}$ 和 $\mathrm{H}_{60}$ 的稳定化能量为

$$
\begin{gathered}
\Delta E-E\left(\mathrm{C}_{60} \mathrm{H}_{60}\right)-E\left(\mathrm{C}_{60}\right)-E\left(\mathrm{H}_{60}\right) \\
-8.931 \text { a. u. }
\end{gathered}
$$

所以, 从分子总能量角度看, 由 $\mathrm{C}_{60}$ 和 $\mathrm{H}_{60}$ 形 成 $\mathrm{C}_{60} \mathrm{H}_{60}$ 时, 使 $\mathrm{C}_{60}$ 簇分子更一步稳定化 了.

由计算出的分子轨道能量, 我们可以得 到, 除去核实电子外, $\mathrm{C}_{60}$ 电子组态为

$$
\begin{aligned}
& \left(1 a_{g}\right)^{2}\left(1 t_{1 u}\right)^{6}\left(1 h_{g}\right)^{10}\left(1 t_{2 u}\right)^{6}\left(1 g_{u}\right)^{8}\left(2 h_{g}\right)^{10} \\
& \left(1 g_{g}\right)^{8}\left(2 t_{1 u}\right)^{6}\left(2 t_{2 u}\right)^{6}\left(2 a_{g}\right)^{2}\left(1 h_{u}\right)^{10}\left(3 a_{g}\right)^{2} \\
& \left(3 t_{1 u}\right)^{6}\left(3 h_{g}\right)^{10}\left(1 t_{1 g}\right)^{6}\left(2 g_{g}\right)^{8}\left(4 h_{g}\right)^{10}\left(4 t_{1 u}\right)^{6} \\
& \left(2 h_{u}\right)^{10}\left(3 t_{2 u}\right)^{6}\left(2 g_{u}\right)^{8}\left(4 t_{2 u}\right)^{6}\left(5 h_{g}\right)^{10}\left(3 g_{g}\right)^{8} \\
& \left(1 t_{2 g}\right)^{6}\left(3 g_{g}\right)^{8}\left(4 g_{u}\right)^{8}\left(6 h_{g}\right)^{10}\left(3 h_{z}\right)^{10}\left(4 g_{g}\right)^{8} \\
& \left(7 h_{g}\right)^{10}\left(4 h_{u}\right)^{10}
\end{aligned}
$$

而 $\mathrm{C}_{60} \mathrm{H}_{60}$ 的电子组态为

$$
\begin{aligned}
& \left(1 a_{g}\right)^{2}\left(1 t_{1 u}\right)^{6}\left(1 h_{g}\right)^{10}\left(1 t_{2 u}\right)^{6}\left(1 g_{u}\right)^{8}\left(2 h_{g}\right)^{10} \\
& \left(1 g_{g}\right)^{8}\left(2 a_{g}\right)^{2}\left(2 t_{2 u}\right)^{6}\left(2 t_{1 u}\right)^{6}\left(3 t_{2 u}\right)^{6}\left(1 h_{u}\right)^{16}
\end{aligned}
$$


表 $1 \mathrm{C}_{60}$ 和 $\mathrm{C}_{60} \mathrm{R}_{60}$ 的前线轨道能量及其间隙(单位: $\mathrm{eV}$ )

\begin{tabular}{|c|c|c|c|}
\hline 分 子 & номо & LUMO & $\Delta E_{H L}$ \\
\hline $\mathrm{C}_{60}$ & $-8.55\left(4 h_{x}\right)$ & $-0.14\left(5 t_{1 n}\right)$ & 8.41 \\
\hline $\mathrm{C}_{60} \mathrm{H}_{60}$ & $-10.48\left(6 h_{u}\right)$ & $8.35\left(6 t_{1 E}\right)$ & 18.83 \\
\hline $\mathrm{C}_{60} \mathrm{~F}_{60}$ & $-13.29\left(12 h_{*}\right)$ & $3.91\left(t_{1 \ldots}\right)$ & 17.20 \\
\hline $\mathrm{H}_{60}$ & $-10.09\left(1 h_{u}\right)$ & $-4.15\left(t_{1.1}\right)$ & 5.94 \\
\hline
\end{tabular}

$\left(3 a_{g}\right)^{2}\left(2 h_{u}\right)^{10}\left(3 h_{g}\right)^{10}\left(1 t_{1 g}\right)^{6}\left(2 g_{g}\right)^{8}\left(3 t_{1 u}\right)^{6}$

$\left(2 g_{u}\right)^{8}\left(3 h_{u}\right)^{10}\left(4 t_{2 u}\right)^{6}\left(4 h_{g}\right)^{10}\left(4 t_{1 u}\right)^{6}\left(3 g_{u}\right)^{8}$

$\left(3 g_{g}\right)^{8}\left(1 t_{2 g}\right)^{6}\left(4 g_{g}\right)^{8}\left(5 h_{g}\right)^{10}\left(4 h_{u}\right)^{10}\left(4 g_{u}\right)^{8}$

$\left(5 t_{1 u}\right)^{6}\left(6 h_{g}\right)^{11}\left(5 t_{2 u}\right)^{6}\left(5 h_{u}\right)^{10}\left(2 t_{1 g}\right)^{6}\left(7 h_{g}\right)^{10}$

$\left(2 t_{28}\right)^{6}\left(5 g_{u}\right)^{8}\left(5 g_{8}\right)^{8}\left(6 h_{u}\right)^{10}$

至于 $\mathrm{C}_{60} \mathrm{~F}_{60}$ 共有 660 个价电子，基态占有 6 个 $a_{g}$ 对称性 $\mathrm{MO}, 32$ 个 $\mathrm{t}$ 对称性 MO, 22 个 $g$ 对称性 MO 和 28 个 $h$ 对称性 MO, 共占有 330 个最低 MO. 为节省篇幅, 只给出最高几 个占有轨道组态

$$
\cdots\left(11 g_{s}\right)^{8}\left(11 g_{u}\right)^{8}\left(16 h_{z}\right)^{10}\left(12 h_{u}\right)^{10} \text {. }
$$

在表 1 中列出各分子的前线 MO 数 值及其 间能隙值.

显然,表 1 中数据进一步说明, 由 $\mathrm{C}_{60}$ 和 $\mathrm{H}_{60}$ 或 $\mathrm{F}_{60}$ 形成 $\mathrm{C}_{60} \mathrm{H}_{60}$ 或 $\mathrm{C}_{60} \mathrm{~F}_{60}$ ，相应的HOMO 均大为降低,而 LUMO 则显著升高. 同时,

$$
\begin{aligned}
& \Delta E_{\mathrm{HL}}\left(\mathrm{C}_{60} \mathrm{H}_{60}\right) \approx \Delta E_{\mathrm{HL}}\left(\mathrm{C}_{60} \mathrm{~F}_{60}\right) \\
& \gg \Delta E_{\mathrm{HL}}\left(\mathrm{C}_{60}\right),
\end{aligned}
$$

这再次表明 $\mathrm{C}_{60} \mathrm{H}_{60}$ 和 $\mathrm{C}_{60} \mathrm{~F}_{60}$ 均比 $\mathrm{C}_{60}$ 分子更 稳定.

\section{考文 㓓}

[1] Kroto, H.W. et al., Nature, 318(1985), 162.

[2] Newton, M.D. et al., J.Am. Chem. Soc., 108 (1986), 2469.

[3] Disch, R.L. et al., J. Chem. Phys. Lett., 125 (1986), 465 .

[4] Shibuya, Tai-chi et sl., Chem. Phys. Lett., 137(1987), 13.

[5] Jun Li et al., Chinese Science Buletin, 35 (1990),2:157.

[6] Feng, J.K. et al., Int. J. Qwont. Chem., 37 (1990),599.

王志中 沈尔忠 王利群 (吉林大学理论化学研究所, 长春 130023)

\section{河外喷流进动的一种可能机制}

不少从活动星系核心发出的成双喷流呈 现反对称的形状，如同字母 S. 这表明喷流 可能在绕着某一轴线作进动.一般认为喷流 是沿着星系核内黑洞的自转轴发出的. 这就 意味着是黑洞在进动. 本文考虑一种可能的 机制，即黑洞进动是由其周围的一个歪斜吸 积盘的外围部分驱动的. 这个模型最初是在 文獸 [1]中针对河内天体 SS433 提出的.

按照文献 [1],吸积盘中能造成黑洞最大 进动的部分是在下述径泃距离处:

$$
r_{p}-\left(\frac{J v_{r}}{\dot{M} v_{\phi}}\right)^{1 / 2}
$$

